\title{
Accuracy of Digital vs. Conventional Implant Impressions
}

\author{
Sang J. Lee, DMD, MMSc ${ }^{1}$, Rebecca A. Betensky, Ph.D. ${ }^{2}$, Grace E. Gianneschi, DMD \\ Candidate. ${ }^{1}$, and German O. Gallucci, DMD, Dr. Med. Dent. Ph.D. ${ }^{1}$ \\ ${ }^{1}$ Department of Restorative Dentistry and Biomaterials Science, Harvard School of Dental \\ Medicine, Boston, MA, USA \\ ${ }^{2}$ Department of Biostatistics, Harvard School of Public Health, Boston, MA, USA
}

\begin{abstract}
The accuracy of digital impressions greatly influences the clinical viability in implant restorations. The aim of this study is to compare the accuracy of gypsum models acquired from the conventional implant impression to digitally milled models created from direct digitalization by three-dimensional analysis.

Thirty gypsum and 30 digitally milled models impressed directly from a reference model were prepared. The models were scanned by a laboratory scanner and 30 STL datasets from each group were imported to an inspection software. The datasets were aligned to the reference dataset by a repeated best fit algorithm and 10 specified contact locations of interest were measured in mean volumetric deviations. The areas were pooled by cusps, fossae, interproximal contacts, horizontal and vertical axes of implant position and angulation. The pooled areas were statistically analysed by comparing each group to the reference model to investigate the mean volumetric deviations accounting for accuracy and standard deviations for precision. Milled models from digital impressions had comparable accuracy to gypsum models from conventional impressions. However, differences in fossae and vertical displacement of the implant position from the gypsum and digitally milled models compared to the reference model, exhibited statistical significance ( $\mathrm{p}<0.001, \mathrm{p}=0.020$ respectively).
\end{abstract}

\section{Introduction}

A gypsum cast poured from a physical impression taken with an elastomeric impression material has been the gold standard in the fabrication of implant restorations. The accurate transfer of the position and angulation of the implants is a critical factor for achieving a precisely fitting prosthesis (Karl et al., 2004). Various techniques and materials have been described to improve the accurate transfer of the position of implants in the clinical situation to the laboratory (Chandran et al., 2010). The findings varied depending on the type of implants, implant-abutment configurations, closed or open tray impression techniques, impression materials, etc. (Spector et al., 1990; Lee et al., 2008; Sorrentino et al., 2010). An

Corresponding Author. Sang J. Lee, DMD, MMSc., Division of Regenerative and Implant Sciences, Harvard School of Dental Medicine. 188 Longwood Avenue, Boston, MA-02115. sang_lee@ hsdm.harvard.edu.

The author(s) declare no potential conflicts of interest with respect to the research, authorhsip, and/or publication of this article. 
inaccurate transfer of the implant position from a physical impression to a gypsum cast may be caused by shrinkage and distortion of the impression materials as well as unstable repositioning of the analog during the laboratory process (Christensen, 2008).

With current CAD/CAM techniques, digital technologies once limited to the scanning and milling of tooth-supported fixed prostheses can now support the implant impression process. Direct intraoral scanning of an implant can create not only a 3-dimensional (3D) virtual model to design and mill the restoration but also fabricate a milled model with a removable implant analog. A digital impression can be created in one of two ways (Guth et al., 2013a): via direct intra-oral scanning or indirectly via scanning casts made from conventional impressions. If scanned directly from the mouth, digital data can be sent electronically to a milling unit that uses this data to fabricate a digital model, thus, intermediate steps involved in conventional impression taking are bypassed.

Accuracy is described by precision and trueness (ISO 5725-1). Precision represents the degree of reproducibility between repeated measurements. Trueness describes the closeness to the actual dimensions of the object. In previously published studies, linear distance measurements were used to investigate the trueness of dental models (Larson et al., 2002; Brosky et al., 2003; Stimmelmayr et al., 2012a; Stimmelmayr et al., 2012b; Stimmelmayr et $a l ., 2012 \mathrm{c}$ ) however, this method is limited by the lack of clear reference points and the inability to measure repeatable reference points. Another method to investigate the accuracy of dental models was attempted by measuring surface points with high trueness by coordinate measuring machines (CMMs) (DeLong et al., 2003). Due to the inability of CMMs to scan in interproximal areas and fissure lines, the geometric size and shape of the testing object was limited and dissimilar from the typical morphology of teeth or the dental arch.

Considering the limitations of these previously utilized measurements of accuracy, a different approach was developed. In order to compare the accuracy of the digital and conventional workflows, a reference scanner was used to capture the surface tessellation language (STL) datasets of the models created via each method and of the original master typodont model from which both types of impressions were taken (Ender \& Mehl, 2013; Guth et al., 2013a; Guth et al., 2013b). Comparing the STL datasets from each workflow to the STL from the original model allows for comparison of the accuracy and trueness of each technique. Comparison is made possible by superimposing each model scan with the master scan individually using a best-fit algorithm.

Previously published studies (Ender \& Mehl, 2013; Guth et al., 2013a; Guth et al., 2013b) have compared digital impressions with conventional impressions but have not compared the accuracy of digitally milled models involving the impression of a dental implant. The aim of this in vitro study is to compare the accuracy of digitally milled models created from direct digitalization to gypsum models acquired from the conventional impression technique. The null hypothesis is that there will be no significant difference in accuracy between the digitally milled models and the gypsum models derived from the conventional impression technique for a single implant restoration. 


\section{Materials and Methods}

The methods of the conventional and digital impression were as described in a previous study (Lee \& Gallucci, 2013). A customized maxillary model (Models Plus, Kingsford Heights, Ind) containing a single implant (Bone Level, Regular Crossfit; Straumann, Basel, Switzerland) located in the maxillary left second premolar position was prepared and used as a reference model. Thirty participants performed the closed tray conventional impression procedure with a vinyl polysiloxane material (Aquasil Ultra Monophase/LV; Dentsply, York, Pa) and the digital impressions with a digital impression system (i-Tero; Cadent iTeroTM, Carstadt, NJ) with the use of a scannable abutment (Scanbody; Straumann, Basel, Switzerland). After repositioning the implant analogue (Straumann, Basel, Switzerland) into the implant impression coping embedded in the impression, the conventional impression was poured with scannable type IV gypsum (GC Fujirock EP OptiXscan, GC America, Alsip, IL, USA) followed by the manufacturer's instructions. Then the conventional models were stored in the room temperature of $21^{\circ} \mathrm{C}$ for at least 96 hours until the expansion of gypsum completed. The digitally milled model was manufactured in a custom milling center (Carstadt, NJ) from STL files generated from the digital impression system.

\section{D dataset generation and analysis}

Fig. 1 represents the study procedure. Scannable abutments were used to digitize the position and angulation of the implant. The reference model, 30 gypsum, and 30 digitally milled models were scanned by a laboratory scanner (Lava Scan ST, 3M ESPE, Seefeld, Germany) with scannable abutments on the implants at the same time. Thirty paired STL datasets describing the surface from the mesial of maxillary left first premolar to the distal of the maxillary left first molar were obtained. All STL datasets from reference, gypsum and digitally milled models were imported into the inspection software (Geomagic Qualify 12.0; Geomagic; Morrisville, NC, USA). To ensure a precise superimposition, irrelevant areas such as below the preparation line and beyond the field of interest were removed. Then, the reference model was set as the reference dataset and the 3D coordinate axes were defined. The STL datasets from the gypsum and the digitally milled models were aligned to the reference dataset by a repeated best fit algorithm based on the selected surfaces of the maxillary left first premolar and molar. The divergences in the $\mathrm{x}-, \mathrm{y}-$, and $\mathrm{z}-$ axes between each reference and test dataset at 10 specified contact locations were measured. The 10 specified areas of interest were located in the horizontal and vertical axes as follows: the top of the scannable implant abutment, the distal and mesial interproximal contact points of the implant site, buccal and mesiobuccal cusps, palatal and mesiolingual cusps, distal and mesial fossae of the first premolar and first molar, respectively. The size of specified locations of analysis was $1 \mathrm{~mm} \times 1 \mathrm{~mm}$ and the divergences were measured in a uniform position in the reference coordinate axes. Divergences between the reference dataset and a test dataset were given as mean positive or negative values and standard deviation in relation to the reference dataset. For each analysis, the overall RMS (root mean square) error and average error were calculated. The three-dimensional differences between a test and the reference were illustrated in a color-coded gradient (Fig. 2). 
The outcomes of divergences measured in the specified locations of interest were pooled by cusps, fossae, interproximal contacts and horizontal and vertical axes of implant position and angulation. The absolute volumetric deviations from the reference model to the gypsum and digitally milled models were calculated after the surfaces were aligned by the best-fit algorithm. A statistical analysis was performed with SAS (SAS Institute Inc.; Cary, NC, USA) to investigate the volumetric deviations from the two comparisons. The repeated observations for each participant at each location were averaged. Separate linear regression models were then fit to the averaged differences in measurements between the conventional and digital impressions for each location, with the only terms in the model being the difference in root mean squared error of the conventional and digital impressions and an intercept. The adequacy of the model was assessed through visual inspection of the histogram of the Pearson standardized residuals and its proximity to standard normal.

\section{Results}

After the best-fit algorithm between the reference model and gypsum or digitally milled models, the positive and negative average volumetric deviation from the gypsum and digitally milled models to the reference model were calculated by pooled locations (Table I, Fig. 3). Accuracy was presented by the average volumetric deviations from the reference model and precision was the standard deviation of accuracy over 10 repeated measures.

On the basis of the volumetric deviations and the standard deviation of all 30 paired datasets from each group to the reference, the pooled data of buccal and lingual cusps showed negative average deviations and no statistical difference between the gypsum and digitally milled models ( $\mathrm{p}=0.159$ and 0.158 respectively). The average volumetric deviations of interproximal contact areas of the gypsum models and digitally milled models compared to the reference model were $0.005 \mathrm{~mm}$ and $0.001 \mathrm{~mm}$, respectively. The difference of the deviations in interproximal contact areas from both groups was not statistically significant $(\mathrm{p}=0.189)$. The average deviations of the distal and mesial fossae of the first premolar and first molar on the gypsum models and digitally milled models compared to the reference model were $-0.017 \mathrm{~mm}$ and $0.016 \mathrm{~mm}$, respectively. The volumetric deviations of fossae areas from the reference model were statistically different between the gypsum and digitally milled models $(\mathrm{p}<0.001)$.

The average deviations in the horizontal axis of the implant were not statistically significant $(\mathrm{p}=0.226$ ), averaging $0.034 \mathrm{~mm}$ for the gypsum models and $0.011 \mathrm{~mm}$ for the digitally milled models, respectively. However, the average vertical displacement of the implant position was $-0.088 \mathrm{~mm}$ for the gypsum and $0.093 \mathrm{~mm}$ for the digitally milled models, respectively, exhibiting a statistical difference $(\mathrm{p}=0.020)$. The precision was poor in both groups compared to that in other locations with precision averaging $0.044 \mathrm{~mm}$ for the gypsum models and $0.061 \mathrm{~mm}$ for the digitally milled models, respectively.

\section{Discussion}

Digital scanning devices are rapidly developing in many fields of dentistry. (Christensen, 2009) Digital scanning has brought innovative dental treatments, such as computer guided 
implantation in combination with a computed tomography (CT) scan or digital impression system, which allows for CAD-CAM based restorations. Acquiring accurate impressions and transferring intraoral situation are a critical step for fabricating precise dental restorations. Only a few scientific data are available about the accuracy of digital impression system in the literature. Direct digitalization showed higher accuracy compared to the conventional impression taking and indirect digitalization (Guth et al., 2013a). Due to the elastic properties of the impression materials, indirect digitization of the impression was not recommended. (DeLong et al., 2001) Furthermore, the digitization of errors of the impressions was influenced by the shape and the interaction effect with the digitization source. (Quaas et al., 2007; Rudolph et al., 2007; Persson et al., 2009) The digitization source with strong changes of curvature and smooth surface texture showed the largest deviations due to high the surface angles and light reflection from the digitization source to the object. (Persson et al., 2008; Persson et al., 2009)

Three dimensional analysis to know the trueness of complex objects and surfaces is difficult. (DeLong et al., 2003) The superimposition of test and reference datasets by a repeated bestfit algorithm was the best methodological compromise to investigate the accuracy of the objects due to the lack of reference points. (Guth et al., 2013a) Several studies employed the superimposition of 3D datasets and discussed their methodology. (Ender \& Mehl, 2013) The finding were affected by the choice of digitization method, reference scanner, best fit alignment, distribution and number of surface data points, which is not available for the researchers. In this study, one reference scanner with a reproducibility of $0.002 \mathrm{~mm}$ was used to create the $3 \mathrm{D}$ datasets and the number of data points was the same and evenly distributed per resolution to minimize the uncertainty caused from the data capturing and analysis process.

This study is one of the first studies that investigate the accuracy of the gypsum models from conventional implant impression and digitally milled models from digital implant impression. The results indicate that the accuracy between the gypsum models and the digitally milled models is not statistically significant, except fossae and vertical position of the implant. The volumetric deviations calculated in this study were pooled by the clinically relevant locations. The outcome may not be interpreted as the "truth" of the absolute difference of a specific location of one model compared to that of the reference model, but the reported volumetric deviations by pooled locations can describe the comparability of different impression techniques and models based on clinical relevance.

The absolute values of the volumetric deviations describe the proximity of each test dataset in relation to the reference representing the trueness. The positive and negative values of the volumetric deviations describe whether the locations are "over" or "under" the reference. (Guth et al., 2013a) The average volumetric deviations of fossae areas on the gypsum models $(-0.017 \mathrm{~mm})$ and digitally milled models $(0.016 \mathrm{~mm})$ compared to the reference model were statistically different. The fossae areas on the gypsum models were "under" the reference surface, but those on the digitally milled models were "over" the reference model. The fossae areas on the milled models were found to be not as deep and detail as the reference model. Such deviations resulted from the limited scanning highly contoured areas 
and the milling process, which is unable to mill the detailed anatomical areas due to the limited size of milling burs and restricted milling axes.

The average vertical displacement of the implant position exhibited statistical differences ( $\mathrm{p}=0.020$ ) by $-0.088 \mathrm{~mm}$ for the gypsum and $0.093 \mathrm{~mm}$ for the digitally milled models, respectively. The implant analogue in the gypsum models was placed "below" the reference model. Due to the elastic property of the impression materials, the implant analogue tends to be pushed upward when replacing it into the closed tray impression and the vertical position of the analogue is more apical than the reference. Tolerance between the implant and impression copings, implant analogue and abutments range from $0.022-0.100 \mathrm{~mm}$. (Ma et al., 1997). The restorations fabricated from the gypsum models would represent hyperocclusion, which would translate into necessary clinical adjustments. The vertical position of the implant analogue in the digitally milled model was found to be above the reference model due to the friction inside of the socket where the implant analogue was placed. The implant restorations from the digitally milled model can result in the lack of occlusal contacts. The standard deviations were interpreted as the precision of each model from different impression techniques. The precision of the gypsum and digitally milled models is comparable, but there was a difference in reproducing the vertical position of the implant. This can be explained by the numerous potential sources of processing errors during the placement of implant analog rather than the systematical errors from the scanning system.

This study showed that the digitally milled models could reproduce the accuracies equivalent to the gypsum models from conventional implant impression except the fossae and vertical position of the implant. The significance of this study indicates that digitally milled models can be integrated into clinical practice as a part of the digital workflow. The 3D comparison methodology can quantify differences using virtual 3D models and be employed to improve the intraoral scanners in the future.

\section{Conclusions}

Within the limitations of the present in-vitro study it can be concluded that milled models from digital impression are comparable to gypsum models from conventional impression in most anatomical areas except the secondary anatomical areas, such as grooves and fossae, where gypsum models represented more details and prominent anatomy. Vertical displacement of implant position from both impression techniques was statistically significantly different from the master model. The vertical position of the implant in gypsum models was more apical than the master model whereas the vertical position of the implant in milled models was more coronal than the master model.

\section{Acknowledgements}

This study was funded by the ITI Foundation (Basel, Switzerland)-Research Grant No. 733-2010. The author(s) would like to grateflly acknowledge the statistical assistance and collaboration through Harvard Catalyst. 


\section{REFERENCES}

Brosky ME, Major RJ, DeLong R, Hodges JS. Evaluation of dental arch reproduction using threedimensional optical digitization. The Journal of prosthetic dentistry. 2003; 90:434-440. [PubMed: 14586306]

Chandran DT, Jagger DC, Jagger RG, Barbour ME. Two- and three-dimensional accuracy of dental impression materials: effects of storage time and moisture contamination. Bio-medical materials and engineering. 2010; 20:243-249. [PubMed: 21084736]

Christensen GJ. Will digital impressions eliminate the current problems with conventional impressions? The Journal of the American Dental Association. 2008; 139:761-763. [PubMed: 18520000]

Christensen GJ. Impressions are changing: deciding on conventional, digital or digital plus in-office milling. The Journal of the American Dental Association. 2009; 140:1301-1304. [PubMed: 19797561]

DeLong R, Heinzen M, Hodges JS, Ko CC, Douglas WH. Accuracy of a system for creating 3D computer models of dental arches. Journal of dental research. 2003; 82:438-442. [PubMed: 12766195]

DeLong R, Pintado MR, Ko CC, Hodges JS, Douglas WH. Factors influencing optical 3D scanning of vinyl polysiloxane impression materials. Journal of prosthodontics : official journal of the American College of Prosthodontists. 2001; 10:78-85. [PubMed: 11512112]

Ender A, Mehl A. Accuracy of complete-arch dental impressions: a new method of measuring trueness and precision. The Journal of prosthetic dentistry. 2013; 109:121-128. [PubMed: 23395338]

Guth JF, Keul C, Stimmelmayr M, Beuer F, Edelhoff D. Accuracy of digital models obtained by direct and indirect data capturing. Clinical oral investigations. 2013a; 17:1201-1208. [PubMed: 22847854]

Guth JF, Wallbach J, Stimmelmayr M, Gernet W, Beuer F, Edelhoff D. Computer-aided evaluation of preparations for CAD/CAM-fabricated all-ceramic crowns. Clinical oral investigations. 2013b; 17:1389-1395. [PubMed: 22868825]

Karl M, Winter W, Taylor TD, Heckmann SM. In vitro study on passive fit in implant-supported 5unit fixed partial dentures. The International journal of oral \& maxillofacial implants. 2004; 19:30-37. [PubMed: 14982352]

Larson TD, Nielsen MA, Brackett WW. The accuracy of dual-arch impressions: a pilot study. The Journal of prosthetic dentistry. 2002; 87:625-627. [PubMed: 12131884]

Lee H, Ercoli C, Funkenbusch PD, Feng C. Effect of subgingival depth of implant placement on the dimensional accuracy of the implant impression: an in vitro study. The Journal of prosthetic dentistry. 2008; 99:107-113. [PubMed: 18262011]

Lee SJ, Gallucci GO. Digital vs. conventional implant impressions: efficiency outcomes. Clinical oral implants research. 2013; 24:111-115. [PubMed: 22353208]

Ma T, Nicholls JI, Rubenstein JE. Tolerance measurements of various implant components. The International journal of oral \& maxillofacial implants. 1997; 12:371-375. [PubMed: 9197102]

Persson AS, Andersson M, Oden A, Sandborgh-Englund G. Computer aided analysis of digitized dental stone replicas by dental CAD/CAM technology. Dental materials : official publication of the Academy of Dental Materials. 2008; 24:1123-1130. [PubMed: 18336900]

Persson AS, Oden A, Andersson M, Sandborgh-Englund G. Digitization of simulated clinical dental impressions: virtual three-dimensional analysis of exactness. Dental materials : official publication of the Academy of Dental Materials. 2009; 25:929-936. [PubMed: 19264353]

Quaas S, Rudolph H, Luthardt RG. Direct mechanical data acquisition of dental impressions for the manufacturing of CAD/CAM restorations. Journal of dentistry. 2007; 35:903-908. [PubMed: 17980951]

Rudolph H, Luthardt RG, Walter MH. Computer-aided analysis of the influence of digitizing and surfacing on the accuracy in dental CAD/CAM technology. Computers in biology and medicine. 2007; 37:579-587. [PubMed: 16844107]

Sorrentino R, Gherlone EF, Calesini G, Zarone F. Effect of implant angulation, connection length, and impression material on the dimensional accuracy of implant impressions: an in vitro comparative 
study. Clinical implant dentistry and related research. 2010; 12(Suppl 1):e63-e76. [PubMed: 19438937]

Spector MR, Donovan TE, Nicholls JI. An evaluation of impression techniques for osseointegrated implants. The Journal of prosthetic dentistry. 1990; 63:444-447. [PubMed: 2184229]

Stimmelmayr M, Erdelt K, Guth JF, Happe A, Beuer F. Evaluation of impression accuracy for a fourimplant mandibular model--a digital approach. Clinical oral investigations. 2012a; 16:1137-1142. [PubMed: 22009182]

Stimmelmayr M, Guth JF, Erdelt K, Edelhoff D, Beuer F. Digital evaluation of the reproducibility of implant scanbody fit--an in vitro study. Clinical oral investigations. 2012b; 16:851-856. [PubMed: 21647591]

Stimmelmayr M, Guth JF, Erdelt K, Happe A, Schlee M, Beuer F. Clinical study evaluating the discrepancy of two different impression techniques of four implants in an edentulous jaw. Clinical oral investigations. 2012c 


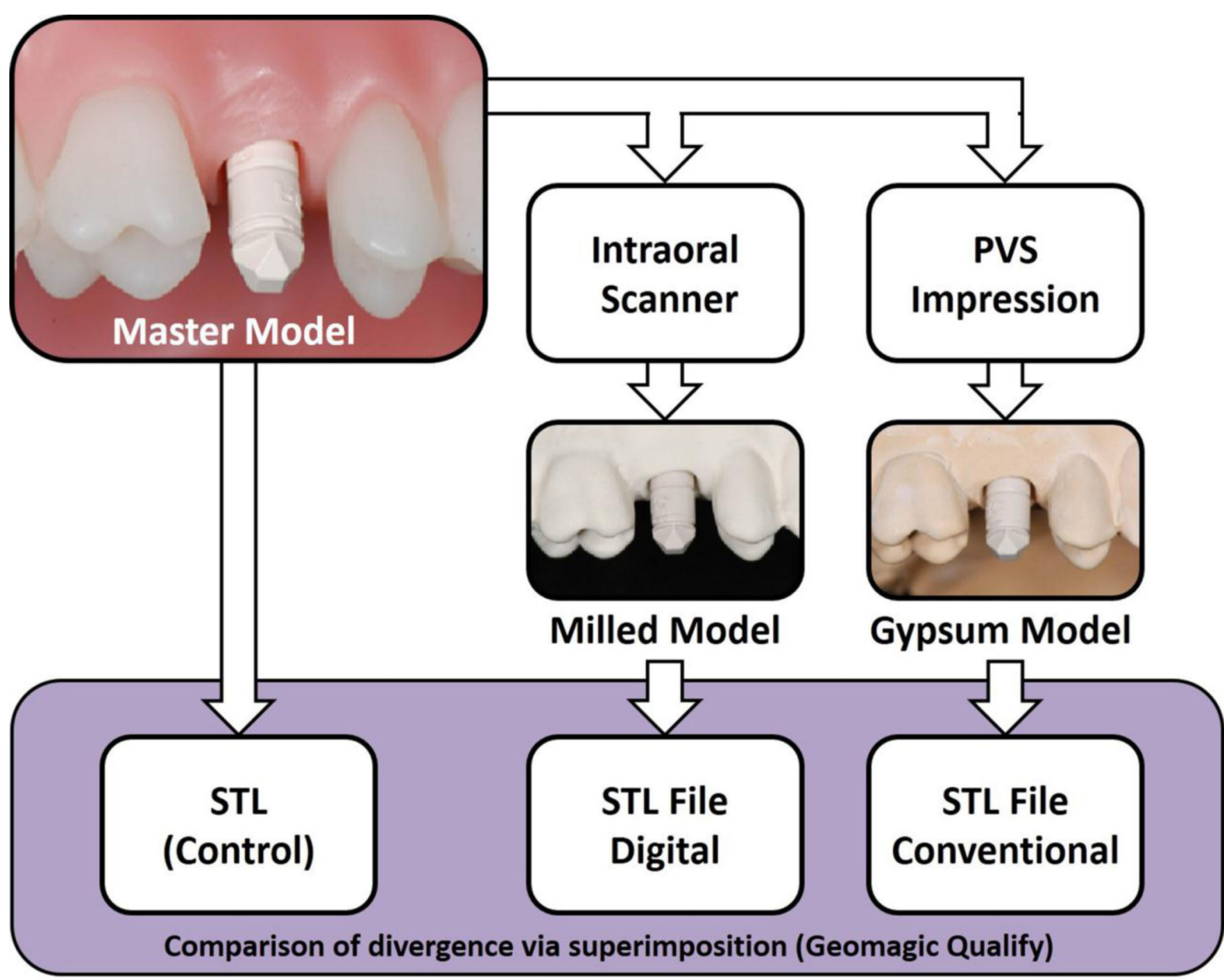

Figure 1.

Impression, model fabrications, 3D dataset generation and analysis for digital and conventional implant impressions workflows. 


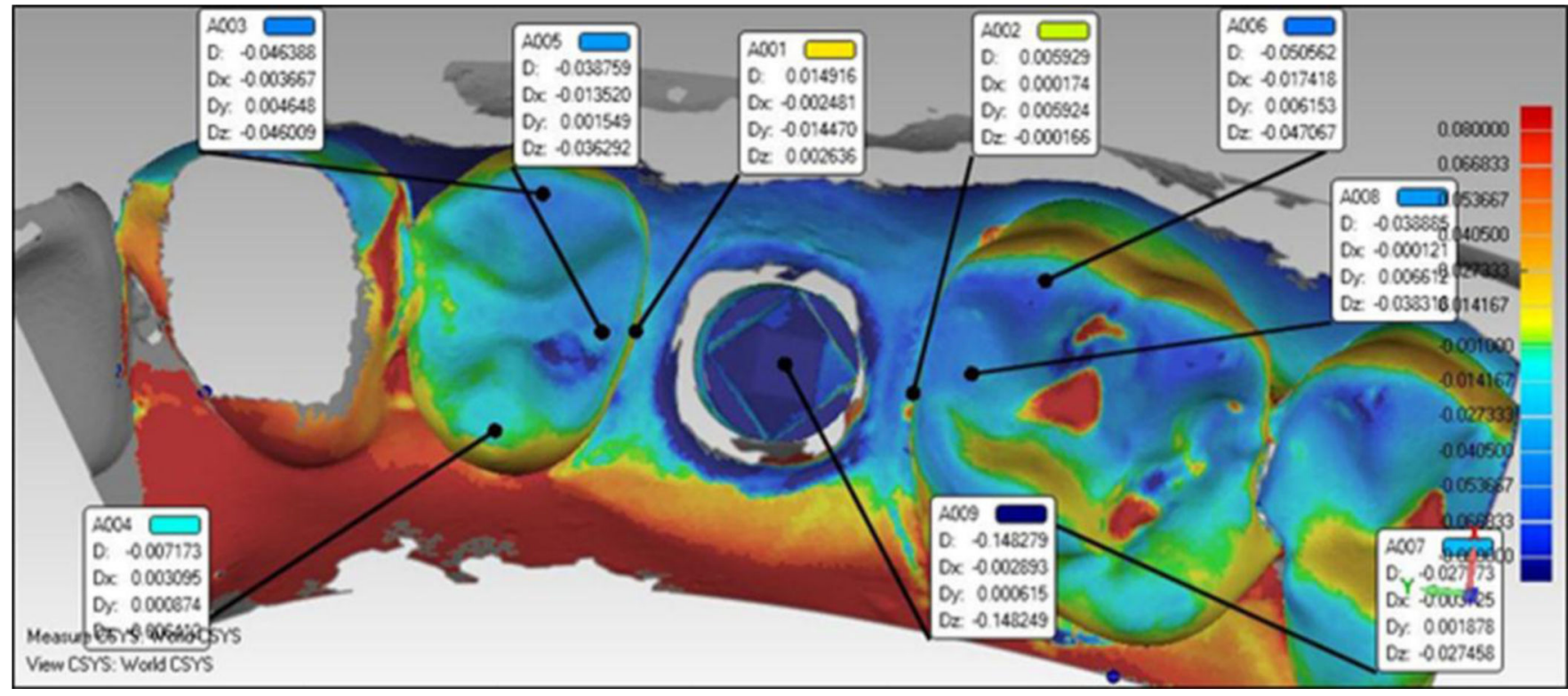

Figure 2.

Three-dimensional differences between a test and the reference STL files after a best fit alignment by an 3D inspection software. Color-coded scale represents the discrepancy of matching. 


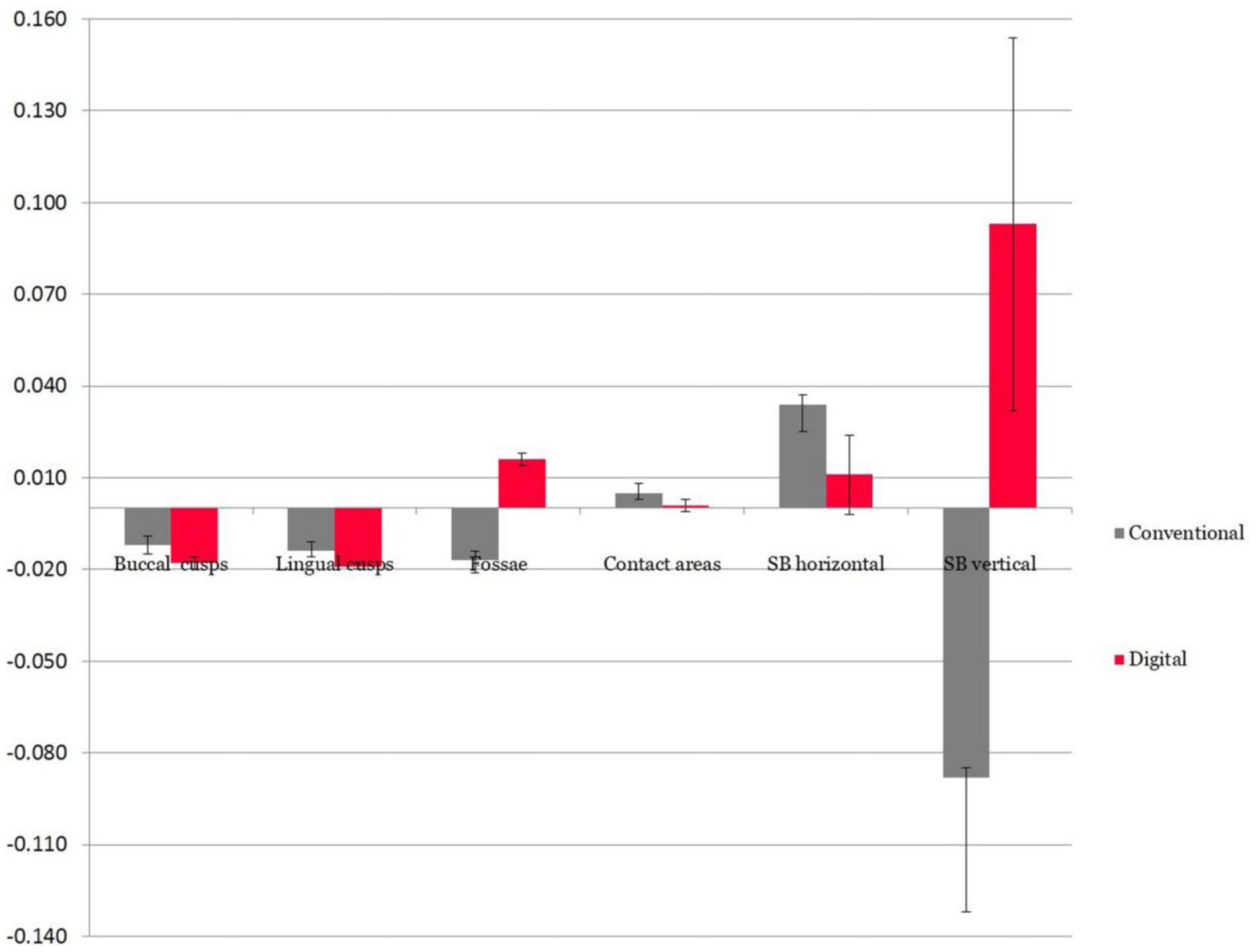

Figure 3.

Positive and negative average volumetric deviation from the gypsum and digitally milled models to the reference model calculated by pooled locations. (In $\mathrm{mm}$ )

SB: scanbody 


\section{Table I}

Volumetric deviations from the gypsum and digitally milled models to the reference model calculated at locations of clinical relevance.

\begin{tabular}{|l|l|l|l|}
\hline Location & Conventional $(\mathbf{m m})$ & Digital $(\mathbf{m m})$ & P value $(<\mathbf{0 . 0 5})$ \\
\hline Buccal cups & $-0.012 \pm 0.003$ & $-0.018 \pm 0.002$ & 0.159 \\
\hline Lingual cusps & $-0.014 \pm 0.002$ & $-0.019 \pm 0.001$ & 0.158 \\
\hline Fossae & $-0.017 \pm 0.004$ & $0.016 \pm 0.002$ & $<0.0001$ \\
\hline Contact areas & $0.005 \pm 0.002$ & $0.001 \pm 0.002$ & 0.189 \\
\hline Scanbody (horizontal) & $0.034 \pm 0.009$ & $0.011 \pm 0.013$ & 0.226 \\
\hline Scanbody (vertical) & $-0.088 \pm 0.044$ & $0.093 \pm 0.061$ & 0.020 \\
\hline
\end{tabular}

